\title{
Is There Still a Place for Continuous Closed Irrigation in the Management of Periprosthetic Total Knee Infection?
}

\author{
Antonio Royo ", Maria Luisa Bertrand, Laura Ramos, Fernando Fernandez-Gordillo and \\ Enrique Guerado
}

Department of Orthopaedic Surgery and Traumatology, Hospital Costa del Sol. University of Malaga, Spain

\begin{abstract}
In recent decades, many technical improvements have been achieved in the use of prosthetic joints, and the risk of infection has been greatly reduced, to current rates of $0.4-2.0 \%$ following primary knee replacement. However, the increasing rate of joint replacements being performed means that the absolute number of such infections remains significant and poses substantial costs to healthcare systems worldwide. Accordingly, further strategies to treat and prevent total joint infections should be investigated.

Infections following knee replacements can compromise the function and durability of arthroplasty. When these infections occur during the immediate postoperative period, irrigation and debridement with component retention can be attempted to salvage the implant. This is an attractive, cheap, low-morbidity treatment for periprosthetic knee infection. However, the results published regarding this procedure are uneven; some studies report the eradication of prosthetic joint infection by debridement alone in $70-90 \%$ of cases but conversely, others have reported a high failure rate for this procedure, averaging $68 \%(61-82 \%)$. The difference could be attributed in part to the multiplicity of variables that may influence the success of the procedure. One such is that of treatment with a continuous irrigation system, which has the theoretical advantage of enabling the administration of antimicrobial agents, as well as the drainage of debris and blood clots.

The objective of this study is to elucidate the overall efficacy of irrigation and debridement with prosthesis retention in infected total knee arthroplasty and to determine whether the addition of a continuous irrigation system influences this efficacy.
\end{abstract}

Keywords: Total knee arthroplasty, periprosthetic infection, irrigation and debridement, continuous irrigation.

\section{INTRODUCTION}

Despite progress in prosthesis science and design, as well as in surgical techniques and perioperative care, infection of the prosthetic knee remains constant in the range of $0.4 \%$ to $2 \%$ during the first year after knee arthroplasty [1-5].

However, although the percentage of patients developing infections after knee replacement surgery is low, the absolute number is reaching intolerable levels, due to the enormous quantity of such operations being performed. Some authors have projected that in the USA the demand for primary knee arthroplasty will grow to 3.48 million patients by 2030 [5]. If $2 \%$ of them develop an infection, as many as 69,000 patients could require treatment for periprosthetic knee infection each year. The potential economic burden to any healthcare system is very considerable and strategies to better treat and to prevent total knee arthroplasty infections should be investigated.

Regarding infection after total knee arthroplasty (TKA), several classification schemes have been proposed and these are used to determine the treatment applied, especially whether the prosthesis should be removed or retained. These

*Address correspondence to this author at the Department of Orthopaedic Surgery and Traumatology, Hospital Costa del Sol, Autovía A-7 Km. 187, 29603. Marbella, Malaga, Spain; Tel: 349519766 69;

E-mail: am_royo@hotmail.com classification systems are mainly based on the clinical presentation, as follows: (a) positive intraoperative culture; (b) early postoperative infection (superficial or deep); (c) late chronic infection; d) acute haematogenous infection [6]. The choice of the most appropriate treatment for a given type of infection after TKA is critical for its control. According to the literature, physicians should only seek to retain the prosthesis in cases of acute infection. The latter may be early postoperative or acute haematogenous infections. By definition, the first of these occurs within one month of implantation, whereas the second is caused by a blood stream seeding the joint from another primary site of infection.

The goal of treatment of an infection at the site of a TKA is eradication of the infection and the restoration of a painless, well-functioning knee. In some cases, the aim is achieved only partially and it is not possible to recover good joint function. Treatment options include chronic antibiotic suppression, irrigation and debridement with component retention, one or two-stage exchange arthroplasty, or salvage procedures such as resection arthroplasty, arthrodesis or even amputation. Of these treatment options, two-stage exchange arthroplasty using an antibiotic-laden cement spacer is considered the gold standard protocol, providing a high rate of infection control, ranging from $85 \%$ to $100 \%$.

However, two-stage revision is associated with pain and limited mobility between stages, and high costs; moreover, it 
can provoke skeletal defects and soft tissue loss, resulting in the possible need for a constrained prosthesis. To avoid these complications, irrigation and debridement has been considered for treating acute postoperative TKA infection.

Rates of infection healing following treatment with prosthesis retention and irrigation plus debridement range from $18 \%$ to $83 \%$ [7-9]. However, direct comparison of infection control rates for different treatment options is difficult because of the many variables that can influence the success of the procedure, such as the exact duration of symptoms after which prosthesis retention is less likely to result in infection eradication (from 2 days [10] to 4 weeks [11]), immunity-impairing host condition, virulence of the infecting organism, implant state, operation history or type of surgery, as well as the absence of randomised, controlled, prospective comparison studies.

One of the variables that could influence the success of the procedure would be the application of a continuous irrigation system, thus enabling the administration of highdose antibiotics and the drainage of debris and blood clots. The literature contains few reports of debridement combined with an associated continuous irrigation system; we found only one other series [12], used in the treatment of septic natural knees, and one concerning case reports [13] on its use for infected TKA (PKI). Therefore, the role of this procedure has yet to be clearly defined.

The purpose of the present study is to determine the overall success of irrigation and debridement with retention of the prosthesis in controlling infection and to examine whether the application of a continuous irrigation system after the surgery improves this success rate. Our operational hypothesis was that irrigation, debridement and the application of a continuous irrigation system after the intervention is likely to provide a better success rate than irrigation and debridement alone; the null hypothesis was that the use of a continuous irrigation system after surgery makes no difference, and the alternative hypothesis was that the use of a continuous irrigation system is associated with a higher failure rate than irrigation and debridement with prosthesis retention alone.

\section{MATERIALS AND METHODS}

\section{Database}

Using our institutional operative database (Doctor, Hewlett Packard, Palo Alto, Ca. USA), all patients with knee prosthetic joint infection undergoing debridement with retention of prosthesis between 1996 and 2010 were identified. We collected 34 patients (10 males and 24 females) with a mean age of 70 years (range, 45-80 years), and followed all of them for a minimum of seven months (mean, 4.39 years; range, 7-136 months). In order to determine the role of a closed continuous irrigation-suction system in the success of the treatment of PKI, we divided the overall series into two historical cohorts: 20 of the 34 patients were treated with irrigation and debridement combined with a continuous closed irrigation-suction system (CCIS), and 14 with irrigation and debridement alone (ID). Twenty-seven patients had undergone a primary total knee arthroplasty and seven a revision arthroplasty for aseptic loosening of knee joints; all of them had negative intraoperative frozen sections and cultures at the time of revision. All patients, in both groups, had received intravenous antibiotics thirty minutes before and immediately following their index knee replacement for a standard period of 24 hours postoperative. All patients had developed either early or acute haematogenous infections, according to the classification of Tsukuyama et al. [1].

\section{Diagnosis}

Periprosthetic infection was diagnosed based on persistent wound drainage, swelling and/or erythema and at least one of the following criteria: (a) isolation of organisms from culture of joint aspirate; (b) abnormal serology, that is, erythrocyte sedimentation rate greater than $30 \mathrm{~mm} / \mathrm{h}$ and a C-reactive protein higher than $1.0 \mathrm{mg} / \mathrm{dL}$; (c) joint fluid white blood cell count more than $2000 / \mu \mathrm{L}$ and neutrophil differential more than $70 \%$. Moreover, we retrospectively reviewed the clinical history of all patients, which is considered the most sensitive method for identifying periprosthetic infection [14].

\section{Surgical Technique}

All patients had fully received the medical and surgical treatment in our hospital. Intraoperative antibiotics were withheld until tissue samples for culture could be obtained for both groups of patients.

\section{Debridement Surgery (ID)}

The knee was approached using the previous incision, debrided with extensive synovectomy and irrigated with at least $9 \mathrm{~L}$ of saline, betadine solution and hydrogen peroxide. The polyethylene component of the total joint was exchanged in $17 \mathrm{knees}$, and the prostheses were confirmed to be stable in all cases. After surgery, suction drains were placed in the patients without a closed irrigation system, for 24 hours (Drenofast CH-14/4,0 min Iberhospitex, S.A. Barcelona, Spain) - this was constituted of a tube with a cross perforated length of $14 \mathrm{~cm}$ connected to a closed wound drainage system with spring bellows suitable for drainage under negative pressure.

\section{Irrigation Suction Continuous System. Surgical Technique (CCIS)}

Once the former procedure was carried out, the suction drain was positioned to perform continuous irrigation. An Argyle thoracic catheter CH-20/6.7 mm (Argyle, Tyco Healthcare Group LP, Mansfield, Massachusetts, USA), was placed in an external suprapatellar location, to function as the inflow tube, and another, the outflow tube, was placed in the arthroscopic anteromedial portal. The inflow tube was connected to a supply of normal saline solution (gravity inflow) and the outflow tube was connected to a suction machine with continuous and vacuum suction. The rate of saline inflow was $6 \mathrm{~L}$ per day.

An infecting organism was identified in 23 patients, based on the results of either pre-operative aspiration or intra-operative cultures. Antibiotics were given under the guidance of an infectious disease specialist, although the choice of the initial antibiotic was empirical unless sensitivities were known from a previous culture. Specific antibiotics were given as soon as sensitivities were known. 
Apart from the antibiotic and the duration of its application, there was no standard procedure, but in most cases $40 \mathrm{gr}$ of gentamicin was added to each two-litre bag of saline solution. The irrigation-suction system was used for two days. The suction drains in the ID group and most of the outflow drains in the CCIS group were removed on the third postoperative day.

\section{Variables}

As stated above, medical records were reviewed and clinical and surgical variables studied to identify those factors known, from prior research, to be predictors of irrigation and debridement outcome, which would act as confounding factors (these were expected to be distributed homogeneously in our series). The clinical risk factors considered were demographic variables (age, sex), duration of symptoms (time to intervention), type of index arthroplasty (primary $v s$ revision) and presentation (early postoperative infection $v s$ later acute haematogenous infection), scored according to American Society of Anesthesiologists recommendations. Surgical risk factors were purulence encountered around the prosthesis, and duration of surgery. The type and methicillin sensitivity of the organism detected by intraoperative culture were also studied as possible predictors of outcome [15-20].

\section{Main Variable}

Outcome variables were identified to define the success or failure of the debridement procedure. Success was considered to be prosthesis retention after treatment with no adverse symptoms, no growth of microorganisms in culture, normalisation of laboratory values and no need for chronic antibiotic treatment. Conversely, treatment failure was defined as the presence of persistent pain, elevated erythrocyte sedimentation rate and C-reactive protein, the need for further surgery, other than irrigation and debridement, in order to control infection, or the need for lifetime antibiotic treatment.

\section{Secondary Variables}

Secondary variables were considered those that could intervene in the outcome, and would act as a confounding variable: age of patient, operative time, timing of irrigation and debridement in relation to the symptoms, length of hospital stay, number of surgical interventions (irrigation and debridement), American Society of Anesthesiologists (ASA) score, gross purulence within the joint. The specific causative organisms were categorised and analysed using organism type as a dichotomous variable, and were first defined as staphylococcus methicillin-sensitive $(\mathrm{n}=12)$ vs all other organisms $(\mathrm{n}=25)$ and then as polybacterial $v s$ monobacterial. Polyethylene component exchange of the total joint was also considered and the overall cost per patient per day calculated.

\section{Statistical Analysis}

Statistical analysis was performed using SPSS Version 11.5 for Windows (SPSS Inc, Chicago, IL, USA). A bivariate analysis of mean values and standard deviations for continuous variables and proportions for categorical variables was performed. The means of continuous variables were compared using the Mann-Whitney (nonparametric) test. Proportions of a categorical outcome were compared using Fisher's exact (nonparametric) test. A P value of less than 0.05 was considered to be significant for a confidence interval of $95 \%$. Due to the small number of patients in our study, a multivariate logistic regression analysis was not performed.

\section{RESULTS}

According to our definitions of successful and failed treatment, ID with prosthesis retention was successful in 25 patients $(73.5 \%)$ and failed in 9 patients $(26.5 \%)$, after the conclusion of follow up. Overall infection rates were similar in both intervention groups, at $75 \%$ in the CCIS and $71.4 \%$ in the control group, with no statistically significant differences between them $(P=1)$.

The patient-specific characteristics were similar in both groups, including during the follow up period. No association was found between health status or host immunity [21] and any treatment group (Tables 1 and 2).

There were no statistically significant differences between the two groups with regard to the number or distribution of infected revision arthroplasties or type of infection, according to the classification of Tsukuyama et al. $(\mathrm{p}=0.672$ and $\mathrm{p}=0.627$, respectively).

Nine patients in our series presented intraoperative purulence around the prosthesis, $(26.47 \%)$ and polyethylene was exchanged in 17 knees (50\%), with no differences between the two treatment groups, $(\mathrm{P}=0.50$ and $\mathrm{P}=0.36$, respectively). There were no differences in outcome, based on the duration of symptoms; for the control group, the median value was 5.5 days, with a difference between the first and the third quartile of 13 days, and for the irrigation system group it was three days, with an interquartile range of 6.75 days $(p=0.507)$. The need for additional surgical debridement procedures to control infection and the number of such interventions were not statistically different between the two groups $(\mathrm{p}=0.562)$.

There were no significant differences $(p=0.704)$ between the two treatment groups regarding those infected with a single organism and those infected with multiple organisms. Twelve knees (35.29\%) were infected with methicillinsusceptible $S$. aureus and there were no differences in this distribution between the two treatment groups $(p=1)$.

Finally, the comparison of the length of stay, for the ID and the CCIS groups, revealed a significant difference: the median hospital stay of patients fitted with a closed system (CCIS) was 22 days $v s 9$ days for ID patients $(\mathrm{p}=0.000)$.

The costs of hospital stay, drugs and other expenses were calculated to be $€ 349$ per day. Since CCIS patients stayed for 13 days longer than ID patients, the extra cost for these 20 CCIS patients was $€ 90,740$.

\section{DISCUSSION}

Two-stage reimplantation for the treatment of TKA periprosthetic infection is currently considered the most effective approach to this major complication. However, the substantial morbidity associated with it must be taken into account. Accordingly, both patients and surgeons tend to view irrigation and debridement with prosthesis retention as 
Table 1. Comparison of Baseline Characteristics and Initial Infection Control Between Both Treatment Groups

\begin{tabular}{|c|c|c|c|}
\hline Variable & $\begin{array}{c}\text { Continuous Closed Irrigation System (CCIS) } \\
(\mathrm{N}=20) \\
\text { Number }(\%)\end{array}$ & $\begin{array}{c}\text { Debridement (ID) } \\
\text { (N=14) } \\
\text { Number }(\%)\end{array}$ & $\begin{array}{c}\text { p-Value } \\
\text { ( Fisher Exact Test ) }\end{array}$ \\
\hline \multicolumn{4}{|l|}{ Gender } \\
\hline Female & $13(65)$ & $11(78.6)$ & \multirow{2}{*}{0.467} \\
\hline Male & $7(35)$ & $3(21.4)$ & \\
\hline \multicolumn{4}{|l|}{ Charlson Comorbidity Index } \\
\hline Low Comorbidity ( 1 or 2 points) & $12(60)$ & $4(28.6)$ & \multirow{2}{*}{0.92} \\
\hline High Comorbidity (>3 points) & $8(40)$ & $10(71.4)$ & \\
\hline \multicolumn{4}{|l|}{ Diabetes Mellitus } \\
\hline Yes & $2(10)$ & $2(14.3)$ & \multirow{2}{*}{1} \\
\hline No & $18(90)$ & $12(85.7)$ & \\
\hline \multicolumn{4}{|l|}{ Rheumatoid arthritis } \\
\hline Yes & $2(10)$ & $0(0)$ & \multirow{2}{*}{0.501} \\
\hline No & $18(90)$ & $14(100)$ & \\
\hline \multicolumn{4}{|l|}{ Infected revision arthroplasty } \\
\hline Yes & $5(25)$ & $2(14.3)$ & \multirow{2}{*}{0.672} \\
\hline No & $15(75)$ & $12(85.7)$ & \\
\hline \multicolumn{4}{|l|}{ Type of Infection } \\
\hline Early Postoperative & $17(85)$ & $13(92.9)$ & \multirow{2}{*}{0.627} \\
\hline Acute Haematogenous & $3(15)$ & $1(7.1)$ & \\
\hline \multicolumn{4}{|l|}{ Microorganism: Staphylococcus Areus } \\
\hline Yes & $7(35)$ & $5(35.7)$ & \multirow{2}{*}{1} \\
\hline No & $13(65)$ & $9(64.3)$ & \\
\hline \multicolumn{4}{|l|}{ Number of microorganisms } \\
\hline Polybacterial & $6(30)$ & $3(21.4)$ & \multirow{2}{*}{0.704} \\
\hline Monobacterial & $14(70)$ & $11(78.6)$ & \\
\hline \multicolumn{4}{|l|}{ ASA Score } \\
\hline I and II & $9(45)$ & $8(57.1)$ & \multirow{2}{*}{0.728} \\
\hline III and IV & $11(55)$ & $6(42.9)$ & \\
\hline \multicolumn{4}{|l|}{ Purulence around prosthesis } \\
\hline Yes & $8(40)$ & $1(7.1)$ & \multirow{2}{*}{0.50} \\
\hline No & $12(60)$ & $13(92.9)$ & \\
\hline \multicolumn{4}{|l|}{ Polyethylene exchange } \\
\hline Yes & $4(20)$ & $13(92.9)$ & \multirow{2}{*}{0.379} \\
\hline No & $16(80)$ & $1(7.1)$ & \\
\hline \multicolumn{4}{|l|}{ Multiple debridements } \\
\hline Yes & $8(40)$ & $3(21.4)$ & \multirow{2}{*}{0.562} \\
\hline No & $12(60)$ & $11(78.6)$ & \\
\hline \multicolumn{4}{|l|}{ Infection control outcome } \\
\hline Controlled & $15(75)$ & $10(71.4)$ & \multirow{2}{*}{1} \\
\hline Not Controlled & $5(25)$ & $4(28.6)$ & \\
\hline
\end{tabular}

an acceptable choice in the setting of acute periprosthetic infection [20].

Seventy-three per cent of the patients in our study achieved infection control and prosthesis retention. Similar high success rates have been reported before [1], for example, Mont et al. reported a success rate of $71 \%$ for late haematogenous infection and $100 \%$ for early postoperative TKA infections without removal of the prosthesis [22]. On the other hand, Silva et al. [11], in their review, reported an 
Table 2. Age of Patients, Duration of Symptons, Hospital Length of Stay, Number of Additional Operations After Irrigation and Debridement and Treatment Duration for Both Treatment Groups

\begin{tabular}{|c|c|c|c|}
\hline Variable & $\begin{array}{c}\text { Continuous Closed Irrigation System (CCIS) } \\
\text { Median (and Interquartile Range) }\end{array}$ & $\begin{array}{c}\text { Debridement (ID) } \\
\text { Mean (and Interquartile Range) }\end{array}$ & $\begin{array}{c}\text { p-Value } \\
\text { (Mann-Whitney U Test) }\end{array}$ \\
\hline \hline Age & $68(11.75)$ & $72.5(7.5)$ & 0.188 \\
\hline Duration of symptoms (days) & $3(6.75)$ & $5.5(13)$ & 0.507 \\
\hline Hospital length of stay* (days) & $22(13.75)$ & $9(9)$ & $0.000^{*}$ \\
\hline Number of interventions & $1(1)$ & $1(1)$ & 0.671 \\
\hline Follow up time (months) & $44(73.25)$ & $40.5(41.5)$ & 0.930 \\
\hline
\end{tabular}

overall failure rate of $67.4 \%$ among infected knee arthroplasties treated with open debridement and prosthesis retention. Nevertheless, in most of the studies included in the latter review, the results of initial surgical treatment were evaluated and the need for any additional intervention was considered to represent a failure of infection control. In our review, repeated debridement was not considered a treatment failure.

We found no statistically significant difference between the two treatment groups with regard to the factors previously identified as being associated with a successful outcome, i.e., young, healthy patients with early postoperative infections, the short-term duration of symptoms, or methicillin-sensitive staphylococcus as the causative organism.

The purpose of this study was to determine the effectiveness of irrigation and debridement combined with the application of a continuous irrigation-suction system, considered to present the following theoretical advantages: diluting the enzymatically active material, diluting the concentration of the causative organism, increasing the efficiency of systemic antibiotics and persistently removing necrotic material.

Certain limitations are inherently present in our study. Data collection of all the variables was performed retrospectively, and so this is not a prospective randomised double blind study and does not provide the same level of evidence. Furthermore, we made no evaluation of clinical function, and so could not reach a clear conclusion as to the effect on the final outcome of multiple interventions required for infection control. These limitations are inherent to all retrospective studies in this field, and further research in which these methodological problems are resolved is needed.

In our study, patients who received open debridement and irrigation combined with the application of a continuous closed irrigation system did not have a different number of operations or a higher or lower success rate, but they did need a longer hospital stay than did patients who received open debridement and irrigation alone (median of 22 days $v s$ 9 days). Therefore, avoiding the use of a closed irrigationsuction system dramatically reduces hospital stay time whilst maintaining the same success rate for infection control.

\section{CONFLICT OF INTEREST}

The authors confirm that this article content has no conflict of interest.

\section{ACKNOWLEDGEMENTS}

Declared none.

\section{REFERENCES}

[1] Tsukayama DT, Estrada R, Gustilo RB. Infection after total hip arthroplasty. A study of the treatment of one hundred and six infections. J Bone Joint Surg Am 1996; 78 (4): 512-23.

[2] Sherrell JC, Fehring TK, Odum S, et al. The Chitranjan Ranawat Award: fate of two-stage reimplantation after failed irrigation and dbridement for periprosthetic knee infection. Periprosthetic Infection Consortium. Clin Orthop Relat Res 2011; 469(1): 18-25.

[3] Meehan AM, Osmon DR, Duffy MCT, et al. Outcome of penicillin-susceptible streptococcal prosthetic joint infection treated with debridement and retention of the prosthesis. Clin Infect Dis 2003; 36 (7): 845-9.

[4] Aboltins CA, Page MA, Buising KL, et al. Treatment of staphylococcal prosthetic joint infections with debridement, prosthesis retention and oral rifampicin and fusidic acid. Clin Microbiol Infect 2007; 13 (6): 586-91.

[5] Garvin KL, Konigsberg BS. Infection following total knee arthroplasty. Prevention and management. J Bone Joint Surg Am 2011; 93 (12): 1167-75.

[6] Tsukuyama DT, Goldberg VM, Kyle R. Diagnosis and management of infection after total knee arthroplasty. J Bone Joint Surg Am 2003; 85-A(Suppl 1): 75-80

[7] Burger RR, Basch T, Hopson CN. Implant salvage in infected total knee arthroplasty. Clin Orthop Relat Res 1991; 273: 105-12

[8] Chiu FY, Chen CM. Surgical debridement and parenteral antibiotics in infected revision total knee arthroplasty. Clin Orthop Relat Res 2007; 461: 130-5

[9] Deirmengian C, Greenbaum J, Lotke PA, et al. Limited success with open debridement and retention of components in the treatment of acute Staphylococcus aureus infections after total knee arthroplasty. J Arthroplasty 2003; 18(Suppl 1): 22-6

[10] Brandt CM, Sistrunk WW, Duffy MC, et al. Staphylococcus aureus prosthetic joint infection treated with debridement and prosthesis retention. Clin Infect Dis 1997; 24 (5): 914-9.

[11] Silva M, Tharani R, Schmalzried TP. Results of direct exchange or debridement of the infected total knee arthroplasty. Clin Orthop Relat Res 2002; 404: 125-31.

[12] Kuo CL, Chang JH, Wu CC, et al. Treatment of septic knee arthritis: Comparison of arthroscopic debridement alone or combined with continuous closed irrigation-suction system. J Trauma 2011; 71 (2): 454-9.

[13] Tsumura H, Ikeda S, Ono T, et al. Synovectomy, debridement and continuous irrigation for infected total knee arthroplasty. Int Orthop 2005; 29 (2): 113-6.

[14] Epine. Available at: http://hws.vhebron.net/epine/Descargas/EPINE $\% 202011 \% 20$ ESPA\%C3\%91A\%20Resumen.pdf [Last accessed: December 2012].

[15] Azzam KA, Seeley M, Ghanem E, Austin MS, Purtl J, Parvizi J. Irrigation and debridement in the management of prosthetic joint infection: Traditional indications revised. J Arthroplasty 2010; 25 (7): 1022-7.

[16] Van Kleunen JP, Knox D, Garino JP, Lee GC. Irrigation and debridement and prosthesis retention for treating acute periprosthetic infections. Clin Orthop Relat Res 2010; 468: 2024-8. 
[17] Choi HR, Van Knoch F, Zurakowski D, Nelson SB, Malchau H. Can implant retention be recommended for treatment of infected TKA? Clin Orthop Relat Res 2011; 469: 961-9.

[18] Odum SM, Fehring TK, Lombardi AV, et al. Irrigation and debridement for periprosthetic infections. Does the organism matter? J Arthroplasty 2011; 26(6 Suppl): 114-8.

[19] Kim YH, Choi Y, Kim JS. Treatment based on the type of infected TKA improves infection control. Clin Orthop Relat Res 2011; 469: 977-84.
[20]

Gardner J, Gioe TJ, Tatman P. Can this prosthesis be saved? Implant salvage attempts in infected primary TKA. Clin Orthop Relat Res 2011; 469: 970-6.

[21] Charlson ME, Pompei P, Ales KL, MacKenzie CR: A new method of classifying prognostic comorbidity in longitudinal studies: development and validation. J Chronic Dis 1987; 40 (5): 373-83.

[22] Mont MA, Waldman B, Banerjee C, Pacheco IH, Hungerford DS. Multiple irrigation, debridement and retention of components in infected total knee arthroplasty. J Arthroplasty 1997; 12 (4): 42633.

(C) Royo et al.; Licensee Bentham Open.

This is an open access article licensed under the terms of the Creative Commons Attribution Non-Commercial License (http://creativecommons.org/licenses/by-nc/3.0/) which permits unrestricted, non-commercial use, distribution and reproduction in any medium, provided the work is properly cited. 